\title{
Review of: "Teriparatide Promotes Bone Healing in Medication-Related Osteonecrosis of the Jaw: A Placebo- Controlled, Randomized Trial"
}

\author{
Vittorio Fusco ${ }^{1}$, Alberto Bedogni ${ }^{2}$, Rodolfo Mauceri ${ }^{3}$, Maura Rossi ${ }^{1}$, Giuseppina Campisi ${ }^{3}$ \\ 1 Azienda Ospedaliera SS Arrigo e Biagio e Cesare Arrigo \\ 2 University of Padua \\ 3 University of Palermo
}

Potential competing interests: The author(s) declared that no potential competing interests exist.

Definitions

Definition of medication-related osteonecrosis of the jaw in 2014

Defined by Salvatore L. Ruggiero et al.

Skeletal-related event (SRE)

Defined by Robert E. Coleman

Double Blind Study

Defined by National Cancer Institute

Randomized Controlled Clinical Trial

Defined by National Cancer Institute

Bisphosphonate-Associated Osteonecrosis of the Jaw

Defined by Sundeep Khosla et al.

Cone-Beam Computed Tomography

Defined by National Cancer Institute

Bias

Defined by John P. A. loannidis

Placebo Control

Defined by National Cancer Institute

Diabetes (Diabetes Mellitus)

Defined by National Human Genome Research Institute (NHGRI)

Antibiotic

Defined by National Cancer Institute

Denosumab

Defined by National Cancer Institute

Osteoporosis

Defined by National Cancer Institute 
Medication related osteonecrosis of the jaw (MRONJ)

Defined by Alberto Bedogni et al.

Sim et colleagues ${ }^{[1]}$ conducted this interesting double-blind randomized controlled trial comparing Teriparatide vs placebo in 34 patients with medication-related osteonecrosis of the jaws diagnosed according to the 2007 ASBMR definition [2]. Patients were randomized to receive either 8 weeks of subcutaneous teriparatide (20 mg/day) or placebo injections (control group), in addition to calcium and vitamin D supplementation and standard clinical care. Participants were observed up to 12 months, both clinically and radiologically with Cone Beam Computed Tomography (CBCT). The authors' conclusion that "Teriparatide improves the rate of resolution of MRONJ lesions and represents an efficacious and safe treatment for if' seems somewhat optimistic.

The issue of teriparatide prescription in cancer patients has been previously raised in the accompanying editorial by Ripamonti et al ${ }^{[3]}$ and it is not the scope of the present review.

Even so, several other aspects of the trial are at issue and would require further clarification.

First, the trial was designed to include 68 participants to allow $80 \%$ power to detect a significant difference between groups, but only half of the desired sample was recruited. Such a limited number of patients could bias the balance of confounders between the study groups despite the random assignment of treatments. Although no statistically significant differences were found overall between the study groups in terms of baseline features, the placebo group included more MRONJ patients with diabetes and ongoing glucocorticoid therapy as compared with the teriparatide group; these factors are likely to worsen MRONJ prognosis and the natural course of disease. In addition, patients in the control group underwent previous MRONJ treatments to a greater extent (surgical therapy and antibiotics), which is likely to be an indirect sign of refractory MRONJ.

Second, the trial enrolled participants based on the diagnosis of

BRONJ as defined by the American Society for Bone and Mineral Research in 2007 [2], literally "the identification of an area of exposed bone in the maxillofacial region that did not heal within 8 weeks after identification by a health care provider, in a patient who was receiving or had been exposed to a bisphosphonate, and had not had radiation therapy to the craniofacial region". Yet, the authors state that also MRONJ patients who had previous exposure to denosumab (DNB) were enrolled in the study. In contrast with nitrogen-containing bisphosphonates (N-BPs), DNB does not bind to hydroxyapatite and incorporate into bone; thus, bone turnover recovers rapidly after drug suspension and might positively influence bone healing of MRONJ lesions. Additional information on the type of Bone Modifying Agent (BMAs) (i.e. BPs or denosumab) used by patients, their distribution between the study groups and the exact number of patients who were still on therapy at the time of recruitment would be necessary to control such potential confounders.

Third, the trial included patients with disseminated cancer bone disease and osteoporosis. This is another potentially confounding factor, as MRONJ shows different aggressiveness and severity in cancer patients taking high dose BMAs for SRE prevention as compared with osteoporosis patients on low dose BMAs. It would be worth knowing the number and the different distribution between groups of primary osteoporosis patients compared with Rheumatoid Arthritis (RA) and/or glucocorticoid induced osteoporosis patients; in fact, these latter are likely to have worse MRONJ prognosis ${ }^{[4][5]}$. 
Fourth, "resolution" of MRONJ lesions, both clinical and radiological, was adopted as the primary study outcome and evaluated by means of oral examination alone and CBCT imaging at enrolment and up to 52 weeks. The term "resolution" was not otherwise defined in the text and it is difficult for the reader to interpret.

It is somewhat surprising that the clinical resolution was strictly followed-up up to one year (monthly up to 3 months and every 3 months afterwords), while the radiological follow-up was limited to the active treatment phase $(0-8 w)$ and then repeated at the conclusion of the study (1 year). One could argue that CBCT was used to monitor jawbone remodelling during teriparatide treatment rather than seek MRONJ radiological resolution.

Fifth, recruitment of ONJ patients was completed between November 2012 and may 2015. This fact may explain the use of an outdated case definition of MRONJ at enrollment, which certainly excluded patients with "bone probing fistulas" from the study. In fact, "bone probing fistulas" were included as a major sign of MRONJ more recently ${ }^{[6]}$. Of note, it is likely that MRONJ patients presenting with "bone probing fistulas" at the end of the study were considered positive outcomes (i.e clinical resolution). This fact could explain the relatively high rate of clinical resolution of MRONJ found in both study arms (45\% vs $33 \%$ ) as compared with the available data on mucosal healing of MRONJ in patients treated non surgically [7][8][9][10][11].

Sixth, in table 1 (Participant Characteristics at Baseline) the authors declare that also stage 0 patients - namely patients without frank bone exposure according to the AAOMS Panel Recommendations on MRONJ[6] - were enrolled in the trial. This is in contrast with the inclusion criteria established for patient recruitment (i.e. bone exposure). Again, clinical resolution was calculated on the number of MRONJ sites rather than the patients. This fact increased the chance to have a positive outcome overall, but did not impact on the number of patients with clinical (and radiological?) resolution (72.2\% in the control group vs. $73.3 \%$ in the teriparatide group), nor improved the quality of life of patients. Seventh, radiological resolution of MRONJ was an intended primary outcome of the study and the authors state that "teriparatide was associated with increased bone volume and thus reduced bony defect size in a greater proportion of patients at 52 weeks". It is difficult for the reader to understand this positive outcome because it is not clearly detailed how they measured the rate of bone volume change on the CBCT images of patients. In addition, "change in MRONJ lesion size" was a specified secondary outcome of the study.

Again, the Authors report that the clinical stage of patients with persistent MRONJ (unresolved bone exposure) was not significantly improved. Provision of data on the "clinical stage" of patients at each time point of the study would be of interest as it could confirm the suggested inconsistency of the AAOMS stage system also used by Sim et al, which is based on the presence of pain and pus discharge that can be only temporarily solved with the use of antibiotics. This condition has been previously named "ping-pong effect" (the migration of patients from Stage 1 to Stage 2, and vice versa) ${ }^{[12]}$.

It is our experience that spontaneous mucosal reparation of a single MRONJ site obtained with non-surgical therapies can be followed by worsening of the underlying jawbone disease, with/without formation of new mucosal sinus track at distant sites and infection episodes at later stages.

We would like to underline that MRONJis definitively a bone disease, rather than a mucosal break disease, and bone imaging (at least CT) should be formally included in the diagnostic work up of the disease not only to diagnose but also to stage and monitor treatment outcomes ${ }^{[12]}$. Unfortunately, the current AAOMS definition ${ }^{[6]}$ also promoted by the 
ASCO/MASCC/ISOO Expert panel on MRONJ ${ }^{[13]}$ still relies on clinical observation of bone exposure (or bone that can be probed) in the oral cavity. Such definition is questionable and already debated in the literature [14][15].

It might be worth considering further cross-analyses using the clinical and imaging data (CBCT) collected by the authors at fixed time intervals $(0,4,8$, and 52 weeks) to confirm the hypothesis that the extent of bone disease does not correlate with the clinical stage. ${ }^{[16]}$.

In conclusion, we aknowledge the authors' scope to increase the level of available evidence on the MRONJ treatment, and agree that teriparatide could be successfully used in selected MRONJ patients. Nonetheless, the generalizability of their study is turned down by the amount of potential confounders that were not addressed, by the absence of a clear definition of the primary outcome, and by an insufficient recruitment of participants.

By providing additional information, as here suggested, they could give additional value to their results.

\section{References}

1. 'le-Wen Sim, Gelsomina L. Borromeo, Claudine Tsao, Rita Hardiman, Michael S. Hofman, Christian Papatziamos Hjelle. (2020). Teriparatide Promotes Bone Healing in Medication-Related Osteonecrosis of the Jaw: A PlaceboControlled, Randomized Trial. JCO. doi:10.1200/jco.19.02192.

2. ${ }^{\text {a, b}}$ Sundeep Khosla, David Burr, Jane Cauley, David W Dempster, Peter R Ebeling, Dieter Felsenberg. (2007). Bisphosphonate-Associated Osteonecrosis of the Jaw: Report of a Task Force of the American Society for Bone and Mineral Research. J Bone Miner Res, vol. 22 (10), 1479-1491. doi:10.1359/jbmr.0707onj.

3. ' Carla I. Ripamonti, Nicola Napoli. (2020). Are We Ready to Use Teriparatide to Treat Medication-Related Osteonecrosis of the Jaw in Clinical Practice?. JCO. doi:10.1200/jco.20.01633.

4. 'O Di Fede, A Bedogni, F Giancola, G Saia, G Bettini, F Toia. (2016). BRONJ in patients with rheumatoid arthritis: a multicenter case series. Oral Dis, vol. 22 (6), 543-548. doi:10.1111/odi.12490.

5. ^Felice S. O'Ryan, Joan C. Lo. (2012). Bisphosphonate-Related Osteonecrosis of the Jaw in Patients With Oral Bisphosphonate Exposure: Clinical Course and Outcomes. Journal of Oral and Maxillofacial Surgery, vol. 70 (8), 18441853. doi:10.1016/j.joms.2011.08.033.

6. a, b, c Salvatore L. Ruggiero, Thomas B. Dodson, John Fantasia, Reginald Goodday, Tara Aghaloo, Bhoomi Mehrotra. (2014). American Association of Oral and Maxillofacial Surgeons Position Paper on Medication-Related Osteonecrosis of the Jaw-2014 Update. Journal of Oral and Maxillofacial Surgery, vol. 72 (10), 1938-1956. doi:10.1016/j.joms.2014.04.031.

7. 'Katia Rupel, Giulia Ottaviani, Margherita Gobbo, Luca Contardo, Giancarlo Tirelli, Paolo Vescovi. (2014). A systematic review of therapeutical approaches in bisphosphonates-related osteonecrosis of the jaw (BRONJ). Oral Oncology, vol. 50 (11), 1049-1057. doi:10.1016/j.oraloncology.2014.08.016.

8. `Salvatore L. Ruggiero, Nina Kohn. (2015). Disease Stage and Mode of Therapy Are Important Determinants of Treatment Outcomes for Medication-Related Osteonecrosis of the Jaw. Journal of Oral and Maxillofacial Surgery, vol. 73 (12), S94-S100. doi:10.1016/j.joms.2015.09.024.

9. ^Ho Kyung Lee, Mi Hyun Seo, Kang Mi Pang, Seung II Song, Jeong Keun Lee. (2013). Comparative Study on Surgical 
and Conservative Management of Bisphosphonate-related Osteonecrosis of the Jaw (BRONJ) in Disease Stage 2. The Journal Of Korean Association of Maxillofacial Plastic and Reconstructive Surgeons, vol. 35 (5), 302-309. doi:10.14402/jkamprs.2013.35.5.302.

10. ^Oliver Ristow, Thomas Rückschloß, Michael Müller, Moritz Berger, Steffen Kargus, Christoph Pautke. (2019). Is the conservative non-surgical management of medication-related osteonecrosis of the jaw an appropriate treatment option for early stages? A long-term single-center cohort study. Journal of Cranio-Maxillofacial Surgery, vol. 47 (3), $491-499$. doi:10.1016/j.jcms.2018.12.014.

11. `Shin-ichi Yamada, Hiroshi Kurita, Eiji Kondo, Shigeru Suzuki, Fumihiro Nishimaki, Nobuhiko Yoshimura. (2018). Treatment outcomes and prognostic factors of medication-related osteonecrosis of the jaw: a case-and literature-based review. Clin Oral Invest, vol. 23 (8), 3203-3211. doi:10.1007/s00784-018-2743-0.

12. ${ }^{a, b}$ A Bedogni, V Fusco, A Agrillo, G Campisi. (2012). Learning from experience. Proposal of a refined definition and staging system for bisphosphonate-related osteonecrosis of the jaw (BRONJ). doi:10.1111/j.1601-0825.2012.01903.x.

13. 'Noam Yarom, Charles L. Shapiro, Douglas E. Peterson, Catherine H. Van Poznak, Kari Bohlke, Salvatore L. Ruggiero. (2019). Medication-Related Osteonecrosis of the Jaw: MASCC/ISOO/ASCO Clinical Practice Guideline. JCO, vol. 37 (25), 2270-2290. doi:10.1200/jco.19.01186.

14. 'Morten Schiodt, Sven Otto, Stefano Fedele, Alberto Bedogni, Ourania Nicolatou-Galitis, Roman Guggenberger. (2019). Workshop of European task force on medication-related osteonecrosis of the jaw-Current challenges. Oral Dis, vol. 25 (7), 1815-1821. doi:10.1111/odi.13160.

15. ^Vittorio Fusco, Daniele Santini, Giuseppina Campisi, Francesco Bertoldo, Gaetano Lanzetta, Toni Ibrahim. (2020). Comment on Medication-Related Osteonecrosis of the Jaw: MASCC/ISOO/ASCO Clinical Practice Guideline Summary. JCO Oncology Practice, vol. 16 (3), 142-145. doi:10.1200/jop.19.00645.

16. `Alberto Bedogni, Stefano Fedele, Giorgio Bedogni, Matteo Scoletta, Gianfranco Favia, Giuseppe Colella. (2014). Staging of osteonecrosis of the jaw requires computed tomography for accurate definition of the extent of bony disease. British Journal of Oral and Maxillofacial Surgery, vol. 52 (7), 603-608. doi:10.1016/j.bjoms.2014.04.009. 